mice showed severely impaired mobilization after administration of G-CSF. In agreement with previous data from an independent group ${ }^{12}$, they found that G-CSF seemed to suppress osteoblast expression of CXCL12 and induced a flattened appearance of endosteal-lining osteoblasts, which was strikingly similar to the appearance of osteoblasts found in Cgt-deficient mice.

As osteoblasts do not express Cgt or G-CSF

receptor, the authors hypothesized that another cell type had to be responsible for G-CSFinduced mobilization. Neurons express G-CSF receptor, and Cgt-deficient mice have several abnormalities resulting from loss of myelin. Previous data had also shown that osteoblasts express $\beta_{2}$-adrenergic receptors, and that bone remodeling requires sympathetic innervation $^{13}$.

Katayama et al. ${ }^{1}$ postulated that because adrenergic stimulation reduces bone formation, an osteoblast-dependent process, insufficient adrenergic stimulation might induce resistance to G-CSF in Cgt-deficient mice. If so, activation of the sympathetic neurons by G-CSF might be responsible (directly or indirectly) for the mobilization phenotype. This view was confirmed using a variety of experimental approaches.

First, the investigators observed the loss of G-CSF-induced mobilization in mice with a chemically damaged sympathetic nervous system. Second, they observed a reduction in mobilization of HSCs in mice treated with a $\beta$-blocker. Finally, they showed that G-CSFinduced mobilization did not occur in mice deficient in an enzyme required for synthesis of norepinephrine, a key sympathetic system neurotransmitter. The lack of mobilization with G-CSF in these mice could be reversed by administration of a $\beta_{2}$-agonist drug, suggest- ing adrenergic stimulation as method of HSC mobilization (Fig. 1).

\section{Nerves in the 'niche'}

Mobilization induced by G-CSF requires 4-6 days of administration and diminishes after 8-10 days despite continued administration. Given this observation, one hypothesis to explain the kinetics of G-CSF-induced mobilization of HSCs might be that osteoblast inhibition only induces mobilization after crossing a functional threshold and cannot be maintained for a prolonged period because of 'desensitization' of the osteoblast $\beta_{2}$-adrenergic receptors, a phenomenon described for many cells expressing these receptors.

Although neurons express G-CSF receptor, it is not clear whether adrenergic neurons are the immediate effector of G-CSF activity. Studies of mobilization in mice with the G-CSF receptor knocked out specifically in the sympathetic system are needed to address this point. Perhaps other nonhematopoietic, non-neurological G-CSF receptor-expressing cells in the bone marrow, such as endothelial cells, are the direct effectors of G-CSF signaling.

Unfortunately, the investigators did not report any microlocalization study of transplanted HSCs in mice, with and without active adrenergic tone, to analyze the specific role of $\beta_{2}$-blocker drugs in localization and retention of HSCs in the bone marrow. In addition, it is not clear whether the mobilization of the hematopoietic progenitors (short-lived and lineage-committed) induced by $\beta_{2}$-agonists also affects more primitive HSCs with self-renewal and multipotential differentiation capabilities. These questions need to be addressed because the results may allow future exploitation of widely used $\beta_{2}$-agonists or $\beta$-blockers for therapeutic mobilization and engraftment of HSCs, respectively.

Such approaches might be important adjuncts for the significant fraction of patients who fail to mobilize sufficient numbers of HSCs after administration of G-CSF or patients who receive a limited number of HSCs such as in cord blood transplantation in adults ${ }^{14}$. New experimental approaches are needed to exploit these mechanisms therapeutically.

G-CSF-dependent mobilization of HSCs through adrenergic stimulation is likely to require other cell communication mechanisms and suggests an even more complex perspective of the 'stem cell niche.' As in smooth muscle cells and the specialized cardiac conductivity system, sympathetic neurons do not establish synapses with every cell. Synchronization and signal amplification to neighboring cells occurs through direct cell-to-cell communication systems. Nature may have provided this system to control the delicate equilibrium between the bone marrow and the peripheral pool of HSCs, and adrenergic drugs may allow us to shift this equilibrium for therapeutic purposes.

1. Katayama, Y. et al. Cell 124, 407-421 (2006).

2. Wilson, A.T.A. Nat. Rev. Immunol. 6, 93-106 (2006).

3. Lord, B.I., Testa, N.G. \& Hendry, J.H. Blood 46, 65-72 (1975).

4. Zhang, J. et al. Nature 425, 836-841 (2003).

5. Calvi, L.M. et al. Nature 425, 841-846 (2003).

6. Kiel, M.J., Yilmaz, O.H., Iwashita, T., Terhorst, C. \& Morrison, S.J. Cell 121, 1109-1121 (2005).

7. Cancelas, J.A. et al. Nat. Med. 11, 886-891 (2005).

8. Lapidot, T., Dar, A. \& Kollet, O. Blood 106, 1901-1910 (2005).

9. Papayannopoulou, T. Blood 103, 1580-1585 (2004).

10. Liu, F., Poursine-Laurent, J. \& Link, D.C. Blood 95 3025-3031 (2000).

11. Levesque, J.P. et al. Blood 104, 65-72 (2004).

12. Semerad, C.L. et al. Blood 106, 3020-3027 (2005).

13. Elefteriou, F. et al. Nature 434, 514-520 (2005).

14. Wagner, J.E. et al. Blood 100, 1611-1618 (2002)

\title{
Launching a leaky vessel
}

A protein that wedges open the gaps between blood vessel cells may contribute to sepsis, suggests a new report (PLoS Med. 3, e46).

In the United States alone, more than 200,000 people each year die from sepsis, in which pathogens infiltrate the bloodstream and induce a systemic inflammatory response. About 40 percent of sepsis patients develop a deadly syndrome in which fluid leaks out of the blood capillaries in the lung and into the alveoli.

Several factors are known to influence the permeability of blood vessels, including angiopoietin-2, a negative regulator of angiogenesis. Samir Parikh et al. found elevated levels of angiopoietin-2 in sepsis patients. The levels of the protein correlated with severity of disease and waned as patients recovered.

The investigators provide evidence that angiopoietin- 2 may be involved in vascular leakage in these patients. For instance, serum from sepsis patients caused gaps to widen between endothelial cells in culture (actin, red; VE-cadherin, an adhesion molecule, green; DNA, blue). Angiopoietin-2 had the same effect on cells, and when administered to healthy mice, caused fluid to build up in their lungs.

Many current approaches to sepsis research focus on targeting the inflammatory response. Targeting regulators of vascular permeability like angiopoietin-2 might offer an alternative, suggest the researchers.

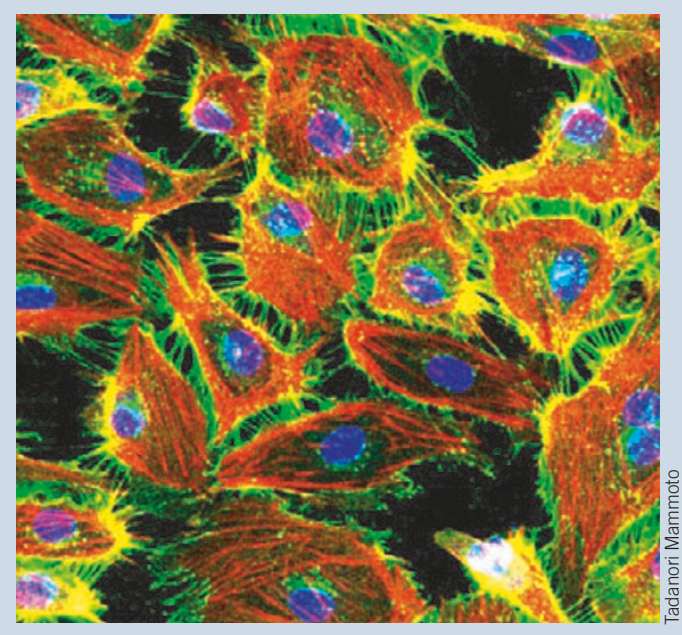

Charlotte Schubert 